\title{
TRANSPARENCIA EN EL SECTOR PÚBLICO A TRAVÉS DE SALAS DE PRENSA ONLINE. GESTIÓN DE RECURSOS COLECTIVOS Y SU INFORMACIÓN EN WEBS MUNICIPALES DE CASTILLA Y LEÓN
}

Public sector transparency through online press rooms. Management of collective resources and information on city websites of Castile and León, Spain

Francisco-Javier Herrero-Gutiérrez, Fernando Martínez-Vallvey, Alejandro Tapia-Frade, Pablo Rey-García y Francisco Cabezuelo-Lorenzo

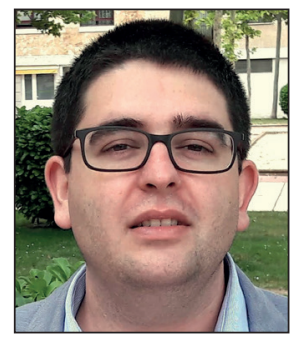

Francisco-Javier Herrero-Gutiérrez es doctor por la Universidad de Salamanca, licenciado en comunicación audiovisual y licenciado en periodismo. Sus principales líneas de investigación son la comunicación deportiva y el estudio de las revistas científicas. Adjunto a la secretaría de redacción de la revista Fonseca, journal of communication. Anteriormente trabajó en diversos medios de comunicación escritos y radiofónicos

http://orcid.org/0000-0002-1362-7406

Universidad de Salamanca, Facultad de Ciencias Sociales Av. Francisco Tomás y Valiente, s/n 37007 Salamanca, España javiherrero82@usal.es

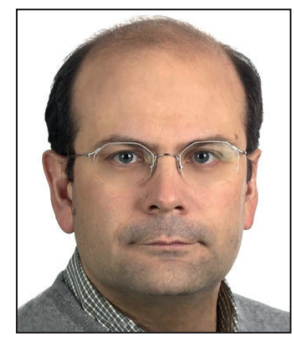

Fernando Martínez-Vallvey es catedrático de Redacción Periodística en la Facultad de Comunicación de la Universidad Pontificia de Salamanca, de la que ha sido decano. Es autor de varias monografías y manuales especializados como Comunicación escrita (Editorial CEF) y Teoría del periodismo (Ed. CEF). Fue durante 10 años director del semanario Tribuna universitaria. Ejerció como periodista en la Cadena Ser y en Tribuna de Salamanca.

http://orcid.org/0000-0001-7686-1323

Universidad Pontificia de Salamanca Compañía, 5. 37002 Salamanca, España fmartinezva@upsa.es

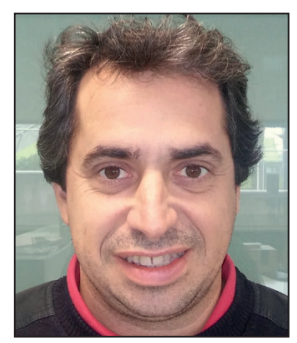

Alejandro Tapia-Frade es licenciado en Publicidad y Relaciones Públicas y doctor en marketing. Autor de unos cuarenta capítulos de libro y artículos en revistas indizadas, está acreditado a profesor contratado doctor y profesor de universidad privada por Aneca y Acsucyl. Es profesor en el Grado de Comunicación en la Universidad Loyola Andalucía. Ha realizado intercambios en Portugal y Alemania.

http://orcid.org/0000-0003-1071-4536

Universidad Loyola de Andalucía Energía Solar, 2. 41014 Sevilla, España ajtapia@uloyola.es

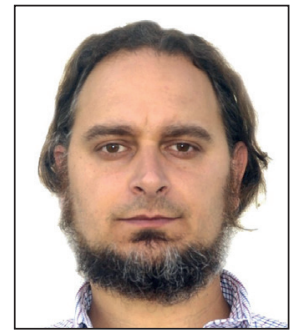

Pablo Rey-García es doctor en Comunicación por la Universidad Pontificia de Salamanca (UPSA), Master en paz, seguridad y defensa por el IUGM-UNED, DEA en Historia Contemporánea por la Universidad de Salamanca, coordinador de relaciones internacionales de la Facultad de Comunicación de la UPSA. Profesor invitado por la Wright State University de Dayton, Ohio, y con estancias de investigación en la Universidad Hebrea de Jerusalén y la Universidad de Lovaina. http://orcid.org/0000-0001-9962-7003 Henry Collet, 90. 37006 Salamanca, España preyga@upsa.es

Artículo recibido el 23-10-2016 Aceptación definitiva: 21-11-2016 


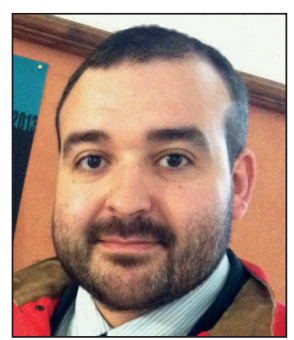

Francisco Cabezuelo-Lorenzo se licenció en Periodismo en la Universidad Complutense de Madrid (UCM), de la cual actualmente es profesor y es licenciado en Publicidad y Relaciones Públicas por la Universidad Camilo José Cela (UCJC). Está acreditado como profesor titular de universidad. Ha participado en varios programas competitivos de I+D+i de convocatorias autonómicas, estatales y europeas.

http://orcid.org/0000-0002-9380-3552

Universidad Complutense de Madrid, Facultad de Ciencias de la Información, Departamento de Periodismo Av. Complutense, s/n. 28040 Madrid, España fcabezuelo@ucm.es

\title{
Resumen
}

Se analiza la transparencia de las webs de los ayuntamientos de Castilla y León (España). A partir de una muestra de 24 municipios -que son las localidades con más de 10.000 habitantes de esta región- se presentan los resultados sobre la gestión e información de los recursos colectivos, así como lo referente a la sala de prensa virtual. Mediante una metodología plenamente contrastada dentro del amplio proyecto del Mapa Infoparticipa, se alcanzan unos resultados especialmente relevantes. Por un lado, la presencia mayoritaria de información sobre la composición de los órganos de gobierno, con diferencias entre los municipios más y menos poblados. Y, por otra parte, se corrobora que la actuación del poder municipal en la región se concreta en la publicación de noticias generalistas y de concesiones, obviando otra serie de información sobre las cuentas o sobre la actuación de la oposición.

\section{Palabras clave}

Información pública; Transparencia; Información municipal; Salas de prensa; Gobierno digital; Ayuntamientos; Sitios web; España.

\begin{abstract}
This research focuses on the transparency of the public websites of the main cities of the region of Castilla y Leon (Spain). Taking as a sample the 24 towns with more than 10,000 inhabitants, this article presents the results related to management and information about the collective resources and the issues concerning the online press room. Using a fully proven methodology developed by a larger project titled Infoparticipa Map, a particularly relevant group of results are achieved. On the one hand, information about the composition of the governing bodies is widely available, with differences between the most and least populated towns. But on the other hand, the performance of municipal power in the region is focused on the publication of general news and concessions to the city governments, obviating other information such as detailed city accounts or the role of the opposition parties.
\end{abstract}

\section{Keywords}

Public information; Transparency; Local information; Press rooms; E-government; City councils; Web sites; Spain.

Herrero-Gutiérrez, Francisco-Javier; Martínez-Vallvey, Fernando; Tapia-Frade, Alejandro; Rey-García, Pablo; Cabezuelo-Lorenzo, Francisco (2017). "Transparencia en el sector público a través de salas de prensa online. Gestión de recursos colectivos y su información en webs municipales”. El profesional de la información, v. 26, n. 3, pp. 421-429.

https://doi.org/10.3145/epi.2017.may.08

\section{Introducción}

La opacidad en política ya no tiene cabida. En los últimos años y con frecuencia creciente los ciudadanos quieren saber qué sucede en su ciudad respecto a la gestión de los recursos. Los ciudadanos buscan información y, entre otros muchos interrogantes, quieren saber qué hacen los políticos electos. Todo esto ha sido causado por

"un creciente debilitamiento de la legitimidad de los gobiernos, las Administraciones y, en general, las instituciones representativas de las democracias contemporáneas" (Villoria-Mendieta; Jiménez-Sánchez, 2012, p. 111).

La transparencia política es un elemento esencial (PiñarMañas, 2014), sobre todo en organismos locales. Los ciudadanos han dejado de ser pasivos en relación a la política en general y a la gestión económica y de los recursos colectivos que hacen los políticos en particular. Las administraciones locales están en el punto de mira de quienes las sustentan; por eso los ayuntamientos se han tenido que poner a trabajar para ser transparentes e informar completa e inteligiblemente de lo que acontece (Moreno-Sardà; Molina-Rodríguez-Navas; Corcoy-Rius, 2013; Molina-Rodríguez-Navas, 2015).

Las políticas de comunicación

"estudian las condiciones jurídicas y políticas que afectan al desarrollo de las libertades públicas" (Rebolledo; Rodríguez-Virgili; Zamora, 2015).

$Y$ es que

"la doctrina del Tribunal Constitucional sobre la libertad de información indica con vehemencia su especial im- 
portancia en la medida en que precondiciona la opinión pública" (Manfredi-Sánchez, 2014).

Por eso es necesario en un Estado social y democrático de derecho

"que los poderes públicos ofrezcan al ciudadano el derecho de poder conocer cómo actúan los poderes públicos, de poder preguntar libremente sobre aquello que le parezca oportuno y de poder formarse su propia opinión" (Manfredi-Sánchez, 2014),

para desembocar todo ello en una opinión pública (Rollnert-Liern, 2015).

Como consecuencia de este movimiento global se creó en 2011 la Alianza para el Gobierno Abierto (Open Government Partnership, OGP) a la que España pertenece.

El 10 de diciembre de 2013 se publicó la Ley 19/2013, de 9 de diciembre, de transparencia, acceso a la información pública y buen gobierno (LTBG) (España, 2013), norma jurídica que nace de la inercia

"histórica, política, social y, por supuesto, jurídica de la emergencia de la transparencia y el acceso a la información" (Cotino-Hueso, 2014, p. 242)

que

"ha permitido considerar el derecho de acceso a la información pública como un derecho fundamental" (Beltrán-Orenes; Martínez-Pastor, 2016, p. 558).

Desde que internet llegó a nuestras vidas, ha sido necesario estar presente en esa gran red de redes. Los ayuntamientos españoles no tardaron en crear sus propias webs de información pública y de promoción turística, consideradas a su vez medios para la visibilidad, notoriedad y reputación, valores en alza en la actual sociedad digital. A pesar de este uso estratégico inicial, legalmente la web, la sede electrónica o los portales de los organismos públicos, son el vehículo a través del que la $L T B G$ manda hacer la publicidad activa a las entidades públicas, incluidas las entidades locales, entre las que se encuentran los ayuntamientos. No es sólo una finalidad, es una obligación que proviene del artículo 5 de la $L T B G$ que especifica la forma concreta en la que llevará a cabo la publicidad activa que recoge la ley:

"La información sujeta a las obligaciones de transparencia será publicada en las correspondientes sedes electrónicas o webs y de una manera clara, estructurada y entendible para los interesados y, preferiblemente, en formatos reutilizables. Se establecerán los mecanismos adecuados para facilitar la accesibilidad, la interoperabilidad, la calidad y la reutilización de la información publicada, así como su identificación y localización" (España, 2013).

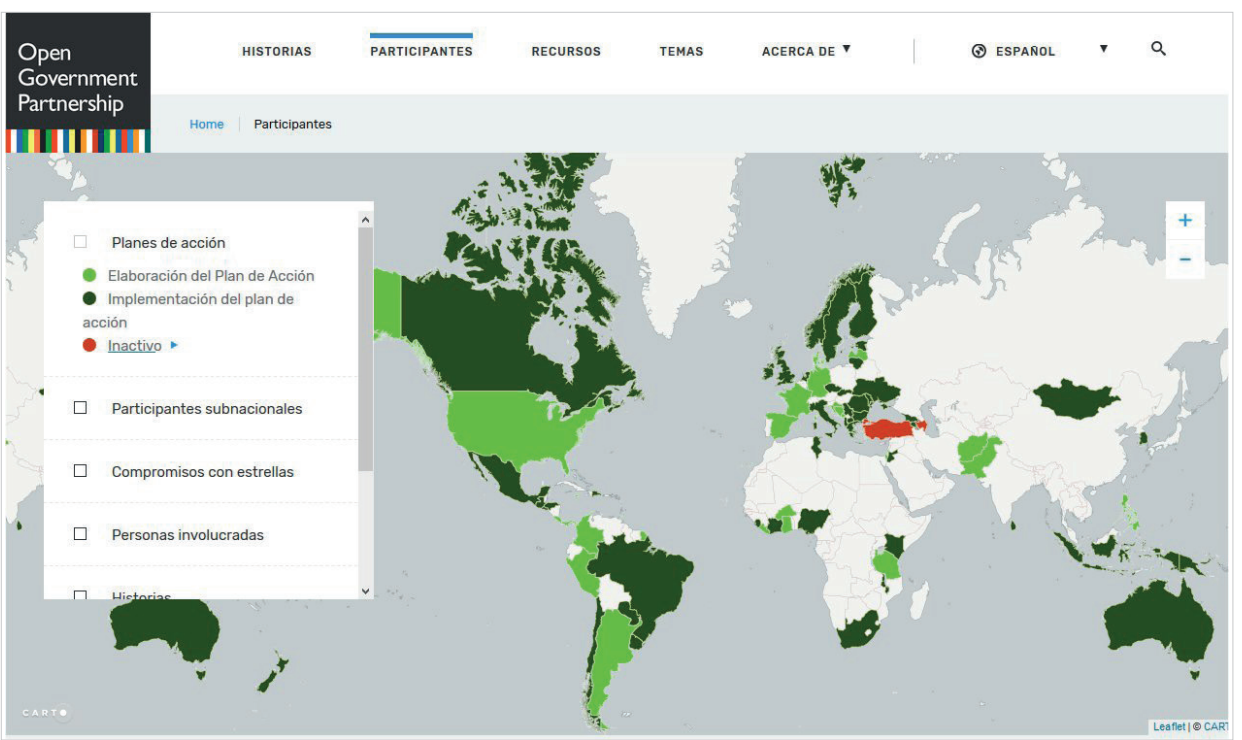

http://www.opengovpartnership.org
Este precepto también está recogido en la Ley 37/2007, de 16 de noviembre sobre reutilización de la información del sector público (España, 2007; 2015).

El presente artículo se engloba dentro de las investigaciones realizadas en el marco del proyecto Comunicación pública, transparencia, rendición de cuentas y participación en los gobiernos locales (Globalcom), con la referencia CSO2013-46997-R, perteneciente al Programa estatal de investigación, desarrollo e 
innovación orientada a los retos de la sociedad del Ministerio de Economía y Competitividad. Este proyecto sigue la filosofía y metodología de otros previos que han abierto el camino en España hacia la investigación en la transparencia informativa, como son los proyectos CSO2012-34687 (20132015) y CSO2015-64568-R (2016-2018) también con financiación ministerial y de los fondos Feder, ambos liderados por la Universitat Autònoma de Barcelona, entre los que destaca como resultado la plataforma del Mapa Infoparticip@, que confirma el impacto que puede y debe tener la investigación científica y académica en la práctica en relación con los ayuntamientos.

\section{http://www.mapainfoparticipa.com}

El Mapa Infoparticip@ proporciona una guía sobre las buenas prácticas que deben incorporarse a las webs, y está poniendo de manifiesto que a medida que se aprende la cultura de la transparencia, las buenas prácticas se extienden rápidamente.

El proyecto CSO2013-46997-R es de especial relevancia para la sociedad porque

"aborda de forma empírica y propositiva la comunicación pública de nuestras entidades locales de forma que se potencien tanto la transparencia y calidad de la información como la rendición de cuentas y la participación ciudadana" (Rebolledo; Rodríguez-Virgili; Zamora, 2015).

En un entorno de creciente digitalización,

"el derecho de los ciudadanos a recibir información de calidad por esta vía parece oportuno para reclamar nuevos espacios de ciudadanía" (Rebolledo; RodríguezVirgili; Zamora, 2015).

Se trata de aprovechar las evaluaciones de las webs para promover que mejore la información que proporcionan y, así, fomentar el derecho al acceso a la información y la transparencia, y atender al principio de publicidad activa (Jurado-Rodas, 2015). El proyecto aboga en su globalidad por que las administraciones se conviertan en fuentes de información transparentes y saneadas, y por empezar a trabajar en la comunicación local como un laboratorio de experiencias.

\section{Metodología}

El objetivo central de este trabajo es conocer el nivel de transparencia en la gestión de los recursos colectivos y su publicación en web en los ayuntamientos de Castilla y León.

Se decidió el uso de la técnica del análisis de contenido. Se considera el método de investigación adecuado, ya que permite conocer la información municipal que los ayuntamientos transmiten de forma objetiva y sin potenciales errores derivados de fallos humanos en la transmisión de información, que podrían producirse con otro tipo de técnicas, como la encuesta.

Se determinó revisar la presencia o ausencia de contenido relativo a las competencias de los órganos de gobierno, su composición y calendario de plenos, acuerdos, reuniones y actividades, planes municipales, los planes de ordenación urbanística, el presupuesto y su gestión, memorias económicas, retribuciones de cargos electos, etc. También se revisó la existencia de noticias y la publicación del perfil del contratante y las concesiones firmadas, acuerdos del pleno municipal, etc.

Para ello se realizó una plantilla de análisis compuesta por 23 variables que estructuran e ilustran las posibilidades desplegadas en web respecto a los elementos mencionados. Estas variables no son arbitrarias. Corresponden a la metodología propia de análisis de la propuesta del Mapa Infoparticip@aprobada en concurrencia competitiva por el ya citado Programa estatal de investigación, desarrollo e innovación orientada a los retos de la sociedad propuesto por el Ministerio de Economía y Competitividad en 2012 para los años 2013-2015, motivo por el que no todas, pero sí la mayoría, encuentran equivalentes con los bloques que la $L T B G$ recoge en su Capítulo II. Publicidad activa (artículos 6 a 8) que no fue publicada hasta el 10 de diciembre de 2013. Hay variables que, sin embargo, no siguen las pautas de la LTBG. Este trabajo, por ejemplo, adjudica la labor de control recogida en el artículo 9 del Capítulo II. Publicidad activa, a la oposición del propio ayuntamiento y no al Consejo de Transparencia y Buen Gobierno, como recoge la Ley, por razones operativas obvias, señalando así una deficiencia de la ley en su concreción en este punto, puesto que el volumen actual de entidades locales haría inviable que un solo organismo llevase el control del cumplimiento de las mismas.

Conforme a los criterios generales inspiradores de la $L T B G$, pero con las variables específicas propias del proyecto Globalcom, se ejecutó dicha plantilla de análisis sobre las webs

Tabla 1. Municipios analizados

\begin{tabular}{|c|c|}
\hline Municipio & Población \\
\hline Valladolid & 306.830 \\
\hline Burgos & 177.776 \\
\hline Salamanca & 148.042 \\
\hline León & 129.551 \\
\hline Palencia & 80.178 \\
\hline Ponferrada (LE) & 67.367 \\
\hline Zamora & 64.423 \\
\hline Ávila & 58.933 \\
\hline Segovia & 53.260 \\
\hline Soria & 39.516 \\
\hline Miranda de Ebro (BU) & 36.724 \\
\hline Aranda de Duero (BU) & 33.065 \\
\hline San Andrés del Rabanedo (LE) & 31.741 \\
\hline Laguna de Duero (VA) & 22.555 \\
\hline Medina del Campo (VA) & 21.274 \\
\hline Benavente (ZA) & 18.879 \\
\hline Villaquilambre (LE) & 18.456 \\
\hline Arroyo de la Encomienda (VA) & 17.572 \\
\hline Santa Marta de Tormes (SA) & 15.011 \\
\hline Ciudad Rodrigo (SA) & 13.975 \\
\hline Béjar (SA) & 13.951 \\
\hline Astorga (LE) & 12.039 \\
\hline La Bañeza (LE) & 10.700 \\
\hline Villablino (LE) & 10.003 \\
\hline
\end{tabular}


Tabla 2. Items de información de recursos colectivos. Bloque 1 (I)

\begin{tabular}{|c|c|c|c|c|c|}
\hline & \multicolumn{4}{|c|}{ Número de habitantes en intervalos } & \multirow[b]{2}{*}{ Total } \\
\hline & Hasta 20.000 & $\begin{array}{c}\text { Entre } 20.001 \text { y } \\
50.000\end{array}$ & $\begin{array}{c}\text { Entre } 50.001 \text { y } \\
100.000\end{array}$ & $\begin{array}{l}\text { Más de } \\
100.000\end{array}$ & \\
\hline & \multicolumn{5}{|c|}{$\%$ de presencia } \\
\hline $\begin{array}{l}\text { ¿Se da información sobre las competencias de los órganos de gobier- } \\
\text { no: pleno, junta de gobierno local y comisiones informativas? }\end{array}$ & 25,00 & 50,00 & 100,00 & 50,00 & 52,17 \\
\hline $\begin{array}{l}\text { ¿Se da información sobre la composición de estos órganos de } \\
\text { gobierno? }\end{array}$ & 55,56 & 50,00 & 80,00 & 100,00 & 66,67 \\
\hline $\begin{array}{l}\text { ¿Se da información sobre el calendario de trabajo de estos órganos } \\
\text { de gobierno? }\end{array}$ & 22,22 & 33,33 & 60,00 & 50,00 & 37,50 \\
\hline $\begin{array}{l}\text { ¿Se publican las convocatorias con los órdenes del día previas a la } \\
\text { celebración de los plenos municipales? }\end{array}$ & 22,22 & 16,67 & 60,00 & 100,00 & 41,67 \\
\hline ¿Se publican las actas del pleno municipal? & 22,22 & 50,00 & 100,00 & 100,00 & 58,33 \\
\hline ¿Se publican los acuerdos del gobierno o de la junta de gobierno local? & 33,33 & 66,67 & 80,00 & 100,00 & 62,50 \\
\hline
\end{tabular}

de los 24 municipios de más de 10.000 habitantes de Castilla y León, que se muestran en la tabla 1.

La recogida de información se efectuó durante el mes de diciembre de 2015. Para la explotación de datos se utilizó el software específico SPSS (Statistical package for social sciences).

\section{Discusión de resultados}

Para la presentación de resultados se decidió realizar el análisis estratificando los resultados en función de la población de los municipios, pues podría parecer prima facie que aquellos municipios con mayor población disponen de mayores recursos materiales y humanos que los más pequeños, aunque hay que señalar que la complejidad en el tratamiento de la información también es mayor. Se consideraron los estratos:

- menos de 20.000 habitantes;

- entre 20.001 y 50.000 habitantes;

- de 50.001 a 100.000 habitantes;

- más de 100.000 habitantes.

\subsection{Gestión e información de los recursos colectivos}

Se analizaron varios items divididos en dos bloques:

- información referente a los órganos de gobierno, en concreto información sobre sus competencias y composición, sus calendarios de trabajo y acuerdos, convocatorias y ac- tas municipales, relación de puestos de trabajo, retribución de cargos electos y ordenanzas municipales;

- información sobre plan de gobierno, plan de actuación municipal, plan estratégico, plan de ordenación urbanística municipal, presupuesto del ayuntamiento y su ejecución, informes de cuentas anuales, memorias de gestión económica, etc.

En el primer bloque, los datos (tabla 2) señalan la presencia mayoritaria de información sobre la composición de los órganos de gobierno en todos los estratos analizados, si bien con mayor frecuencia en los municipios más poblados. Similar tendencia, aunque ya minoritaria en el caso de los municipios menos poblados, puede apreciarse para la existencia de información sobre convocatorias, acuerdos y actas municipales. Sin duda, ésta es una información que requiere de recursos operativos diarios, pues en principio la dinamicidad debe considerarse característica propia de la misma.

A pesar de su presencia minoritaria en los municipios menos poblados (de hasta 20.000 habitantes), debe señalarle una tendencia más errática en el caso de la información sobre las competencias que estos órganos asumen, así como de su calendario de trabajo, si bien es cierto que en este último caso es más frecuente en municipios de más de 50.000 habitantes.

De igual modo, de acuerdo con los datos mostrados en tabla 3, la publicación de una relación con los puestos de trabajo

Tabla 3. Items de información de recursos colectivos. Bloque 1 (II)

\begin{tabular}{|c|c|c|c|c|c|}
\hline & \multicolumn{4}{|c|}{ Número de habitantes en intervalos } & \multirow[b]{2}{*}{ Total } \\
\hline & Hasta 20.000 & $\begin{array}{l}\text { Entre } 20.001 \text { y } \\
50.000\end{array}$ & $\begin{array}{c}\text { Entre } 50.001 \text { y } \\
100.000\end{array}$ & $\begin{array}{l}\text { Más de } \\
100.000\end{array}$ & \\
\hline & \multicolumn{5}{|c|}{$\%$ de presencia } \\
\hline $\begin{array}{l}\text { ¿Se publica la relación de los puestos de trabajo de la cor- } \\
\text { poración y las retribuciones, según las categorías? }\end{array}$ & & 16,67 & 80,00 & 100,00 & 37,50 \\
\hline ¿Se publican las retribuciones de los cargos electos? & 11,11 & 16,67 & 40,00 & 100,00 & 33,33 \\
\hline ¿Se publican las ordenanzas municipales? & 88,89 & 66,67 & 100,00 & 75,00 & 83,33 \\
\hline
\end{tabular}




\begin{tabular}{|c|c|c|c|c|c|}
\hline & \multicolumn{4}{|c|}{ Número de habitantes en intervalos } & \multirow[b]{2}{*}{ Total } \\
\hline & $\begin{array}{l}\text { Hasta } \\
20.000\end{array}$ & $\begin{array}{c}\text { Entre } 20.001 \text { y } \\
50.000\end{array}$ & $\begin{array}{l}\text { Entre } 50.001 \text { y } \\
100.000\end{array}$ & $\begin{array}{l}\text { Más de } \\
100.000\end{array}$ & \\
\hline & \multicolumn{5}{|c|}{$\%$ de presencia } \\
\hline $\begin{array}{l}\text { ¿Se da información sobre el plan de gobierno (PG), plan de actua- } \\
\text { ción municipal (PAM) y / o plan estratégico? }\end{array}$ & 11,11 & 16,67 & 80,00 & 50,00 & 33,33 \\
\hline $\begin{array}{l}\text { ¿Se da información sobre el plan de ordenación urbanística munici- } \\
\text { pal (POUM) u otras normas de planificación urbanística? }\end{array}$ & 88,89 & 83,33 & 100,00 & 100,00 & 91,67 \\
\hline $\begin{array}{l}\text { ¿Se da información sobre otros planes municipales: Agenda21, } \\
\text { juventud, participación ciudadana, etc.? }\end{array}$ & 44,44 & 66,67 & 100,00 & 100,00 & 70,83 \\
\hline ¿Se publica el presupuesto del ayuntamiento? & 55,56 & 16,67 & 100,00 & 100,00 & 62,50 \\
\hline ¿Se publica información sobre la ejecución del presupuesto? & 22,22 & & 80,00 & 50,00 & 33,33 \\
\hline $\begin{array}{l}\text { ¿Se publican los informes anuales de la cuenta general y la memoria } \\
\text { de la gestión económica de la Comisión Especial de Cuentas? }\end{array}$ & & & 40,00 & 100,00 & 25,00 \\
\hline
\end{tabular}

y sus retribuciones es sólo mayoritaria en los estratos más elevados (de más de 50.000 habitantes).

Todavía más restrictiva es la información sobre las retribuciones de cargos electos, siendo minoritaria en todos los estratos salvo el de más de 100.000 habitantes.

En el lado contrario, las ordenanzas municipales son publicadas mayoritariamente en todos los estratos estudiados.

En el segundo bloque, sobre información de tipo operativo, debe considerarse en términos amplios que existe la tendencia antes expuesta: los municipios muestran menos información, quizá debido a la falta de recursos para poder afrontar su revisión y actualización (tabla 4).

Se observa que la provisión de información es total en los dos estratos superiores en relación a los planes urbanísticos municipales, y otros planes como el plan Agenda21, planes de juventud, de participación ciudadana, etc., y también en
Tabla 5. Anova de un factor para los datos de información de recursos colectivos

\begin{tabular}{|l|c|c|c|c|c|}
\hline \multicolumn{1}{|c|}{ Anova } & $\begin{array}{c}\text { Suma de } \\
\text { cuadrados }\end{array}$ & gl & $\begin{array}{c}\text { Media } \\
\text { cuadrática }\end{array}$ & F & Sig. \\
\hline Inter-grupos & 288,375 & 3,000 & 96,125 & 14,110 & 0,000 \\
\hline Intra-grupos & 136,250 & 20,000 & 6,813 & & \\
\hline Total & 424,625 & 23,000 & & & \\
\hline
\end{tabular}

relación con el presupuesto, pero no con su ejecución. Se dice cuánto se va a gastar, pero no se justifica dicho gasto (si bien es cierto que es mucho más frecuente en municipios de más de 50.000 habitantes).

De igual modo, la publicación de informes anuales y memorias de gestión económica es exclusiva en los municipios de más de 50.000 habitantes. Parecida situación se da en relación con la publicación en la web del plan de gobierno, el

Tabla 6. Prueba HSD Tukey para los datos de información de recursos colectivos.

\begin{tabular}{|c|c|c|c|c|c|c|}
\hline \multirow{2}{*}{$\begin{array}{c}\text { (I) Número de habitantes en } \\
\text { intervalos }\end{array}$} & \multirow{2}{*}{$\begin{array}{l}\text { (J) Número de habitantes en } \\
\text { intervalos }\end{array}$} & \multirow{2}{*}{$\begin{array}{l}\text { Diferencia de } \\
\text { medias (I-J) }\end{array}$} & \multirow{2}{*}{ Error típico } & \multirow{2}{*}{ Sig. } & \multicolumn{2}{|c|}{$\begin{array}{c}\text { Intervalo de confianza } \\
\text { al } 95 \%\end{array}$} \\
\hline & & & & & $\begin{array}{l}\text { Límite } \\
\text { superior }\end{array}$ & $\begin{array}{l}\text { Límite } \\
\text { inferior }\end{array}$ \\
\hline \multirow{3}{*}{ Hasta 20.000} & Entre 20.001 y 50.000 & $-0,500$ & 1,376 & 0,983 & $-4,350$ & 3,350 \\
\hline & Entre 50.001 y 100.000 & $-7,000$ & 1,456 & 0,001 & $-11,075$ & $-2,925$ \\
\hline & Más de 100.000 & $-7,750$ & 1,568 & 0,000 & $-12,140$ & $-3,360$ \\
\hline \multirow{3}{*}{ Entre 20.001 y 50.000} & Hasta 20.000 & 0,500 & 1,376 & 0,983 & $-3,350$ & 4,350 \\
\hline & Entre 50.001 y 100.000 & $-6,500$ & 1,580 & 0,003 & $-10,924$ & $-2,076$ \\
\hline & Más de 100.000 & $-7,250$ & 1,685 & 0,002 & $-11,966$ & $-2,534$ \\
\hline \multirow{3}{*}{ Entre 50.001 y 100.000} & Hasta 20.000 & 7,000 & 1,456 & 0,001 & 2,925 & 11,075 \\
\hline & Entre 20.001 y 50.000 & 6,500 & 1,580 & 0,003 & 2,076 & 10,924 \\
\hline & Más de 100.000 & $-0,750$ & 1,751 & 0,973 & $-5,651$ & 4,151 \\
\hline \multirow{3}{*}{ Más de 100.000} & Hasta 20.000 & 7,750 & 1,568 & 0,000 & 3,360 & 12,140 \\
\hline & Entre 20.001 y 50.000 & 7,250 & 1,685 & 0,002 & 2,534 & 11,966 \\
\hline & Entre 50.001 y 100.000 & 0,750 & 1,751 & 0,973 & $-4,151$ & 5,651 \\
\hline
\end{tabular}

La diferencia de medias es significativa al nivel .05 . 
plan de actuación municipal y el plan estratégico, ya que si bien dicha publicación no es exclusiva de los estratos mencionados, hay que señalar escasa presencia en los demás.

Es relevante señalar que se pudo obtener significación estadística en la tendencia antes señalada mediante la realización de un análisis complementario, que se muestra en las tablas 5 y 6.

En conclusión, en relación con los datos analizados puede decirse que los municipios de Castilla y León de más de 50.000 habitantes informan en mayor medida de los recursos colectivos que los municipios más pequeños (de entre 10.000 y 50.000 habitantes).

La publicación de informes anuales y memorias de gestión económica es exclusiva en los municipios de más de 50.000 habitantes

\subsection{La sala de prensa municipal}

Los elementos que se incluyen en este trabajo respecto de la publicación de noticias y otros elementos que debieran constituir parte de una sala de prensa online son la publicación de:

- noticias generalistas;

- noticias sobre las actuaciones de los miembros del gobierno relacionadas con el rendimiento de cuentas;

- noticias sobre las actuaciones de los miembros de la oposición relacionadas con el control de la gestión del gobierno;

- noticias en las que se contrastan las informaciones de miembros del gobierno, de la oposición, y de técnicos en su caso;

- publicaciones del perfil del contratante y de las contrataciones y las concesiones firmadas por la corporación con otras entidades, empresas o particulares;

- si publica en la web el Decálogo de buenas prácticas de la comunicación local pública o algún otro documento similar.

Por ello, sin considerarse exhaustivo, sí que se puede interpretar como sintomático de las actitudes tomadas por las corporaciones municipales respecto de la publicidad que consideran oportuna trasladar a los ciudadanos, aun cuando dichas noticias en algún caso podrían no ser beneficiosas para la corporación o sus integrantes.

En efecto, las corporaciones municipales publican noticias generalistas en la web, lo que a priori podría entenderse como un esfuerzo por parte de los representantes municipales de dar a conocer la situación del municipio (tabla 7).

\section{Los municipios de Castilla y León de más de 50.000 habitantes informan en mayor medida de los recursos colectivos que los municipios de entre 10.000 y 50.000 habitantes}

La misma tendencia puede señalarse cuando la información es referente al perfil del contratante y las concesiones firmadas con otras entidades, empresas o particulares, que también podría entenderse como un esfuerzo del gobierno municipal por mostrar transparencia en sus adjudicaciones.

Sin embargo, la situación cambia cuando las noticias son sobre las actuaciones del gobierno en relación al rendimiento de cuentas, o en noticias sobre los miembros de la oposición en lo relativo al control del gobierno, o en noticias que contrastan las informaciones dadas por el poder municipal. En todos estos casos, la actuación municipal sólo puede considerarse como silencio, siendo además una actitud general en todos los estratos analizados.

\begin{tabular}{|c|c|c|c|c|c|}
\hline & \multicolumn{4}{|c|}{ Número de habitantes en intervalos } & \multirow[b]{2}{*}{ Total } \\
\hline & $\begin{array}{l}\text { Hasta } \\
20.000\end{array}$ & $\begin{array}{l}\text { Entre } 20.001 \\
\text { y } 50.000\end{array}$ & $\begin{array}{l}\text { Entre } 50.001 \\
\text { y } 100.000\end{array}$ & $\begin{array}{l}\text { Más de } \\
100.000\end{array}$ & \\
\hline & \multicolumn{5}{|c|}{$\%$ de presencia } \\
\hline ¿Se publican noticias generalistas en la web? & 88,89 & 100,00 & 100,00 & 100,00 & 95,83 \\
\hline $\begin{array}{l}\text { ¿Se publican noticias sobre las actuaciones de los miembros del gobier- } \\
\text { no relacionadas con el rendimiento de cuentas? }\end{array}$ & 37,50 & & 40,00 & 25,00 & 26,09 \\
\hline $\begin{array}{l}\text { ¿Se publican noticias sobre las actuaciones de los miembros de la oposi- } \\
\text { ción relacionadas con el control de la gestión del gobierno? }\end{array}$ & 11,11 & & 20,00 & 50,00 & 16,67 \\
\hline $\begin{array}{l}\text { ¿Se publican noticias en las que se contrastan las informaciones de } \\
\text { miembros del gobierno, de la oposición, y de técnicos en su caso? }\end{array}$ & 11,11 & & & 25,00 & 8,33 \\
\hline $\begin{array}{l}\text { ¿Se informa del perfil del contratante y de las contrataciones y las } \\
\text { concesiones firmadas por la corporación con otras entidades, empresas } \\
\text { o particulares? }\end{array}$ & 88,89 & 100,00 & 100,00 & 100,00 & 95,83 \\
\hline $\begin{array}{l}\text { ¿Se publica en la web el Decálogo de buenas prácticas de la comunicación } \\
\text { local pública o algún otro documento similar? }\end{array}$ & & 16,67 & 20,00 & 25,00 & 12,50 \\
\hline
\end{tabular}


Es decir, en todos los casos se informa de cuestiones generales y de concesiones firmadas, pero no de las actuaciones municipales sobre la gestión de cuentas, ni de las actuaciones de la oposición en su labor de control del poder municipal, ni evidentemente noticias que pudieran contravenir o contrastar lo que el poder municipal o la oposición han dicho o hecho.

Este silencio, talto de la actuación del gobierno como de la oposición, no ayuda a considerar la hipótesis antes planteada, pues no puede señalarse como una actuación que contribuya a la generación de transparencia ni al establecimiento de relaciones de confianza respecto de los representantes municipales. $Y$ es, como se ha señalado, una situación generalizada.

Con el fin de contrastar si en efecto no pueden establecerse diferencias significativamente relevantes en función del tamaño del municipio en relación con la actuación del poder municipal en la llamada sala de prensa, se realizó un análisis complementario que se muestra en la tabla 8. Los resultados señalan que en efecto no pueden establecerse diferencias significativas en función del tamaño del municipio.

En todos los casos se informa de cuestiones generales y de concesiones firmadas, pero no de las actuaciones municipales en lo relativo a la gestión de cuentas

\section{Conclusiones}

La transparencia del sector público respecto de la publicidad en web de elementos de gestión de los recursos colectivos y su publicación en forma de noticias se aborda en esta investigación desde una doble perspectiva.

Por un lado, la gestión y rendición de cuentas al ciudadano de los recursos colectivos, materializado la presencia de información referente a los órganos de gobierno (por ejemplo, información sobre las competencias de estos órganos, sus calendarios de trabajo, convocatorias, acuerdos y actas, ordenanzas municipales, puestos de trabajo y retribuciones de cargos electos, etc.) y también la presencia de otros elementos que evidencian la gestión de recursos colectivos como la existencia de información sobre el plan de actuación municipal, plan de ordenación urbanística municipal, el presupuesto del ayuntamiento y su ejecución y memorias de gestión económica.

Dichos elementos se consideraron, además, en función de la población del municipio (menos de 20.000 habitantes, entre 20.001 y 50.000 , entre 50.001 y 100.000 , y más de 100.000).

Los resultados indican la presencia mayoritaria de información sobre la composición de los órganos de gobierno en todos los estratos analizados, aunque con mayor frecuencia en los municipios más poblados. Similar tendencia, aunque minoritaria en el caso de los municipios menos poblados, puede apreciarse para la existencia de información sobre
Tabla 8. Anova de un factor para los datos de la sala de prensa municipal

\begin{tabular}{|l|c|c|c|c|c|}
\hline \multicolumn{1}{|c|}{ Anova } & $\begin{array}{c}\text { Suma de } \\
\text { cuadrados }\end{array}$ & gl & $\begin{array}{c}\text { Media cua- } \\
\text { drática }\end{array}$ & F & Sig. \\
\hline Inter-grupos & 3,394 & 3,000 & 1,131 & 1,753 & 0,190 \\
\hline Intra-grupos & 12,258 & 19,000 & 0,645 & & \\
\hline Total & 15,652 & 22,000 & & & \\
\hline
\end{tabular}

convocatorias, acuerdos y actas municipales, puestos de trabajo y retribuciones de cargos electos. Esta tendencia también puede apreciarse en el plan de actuación municipal, plan de ordenación urbanística municipal, presupuesto del ayuntamiento y su ejecución y las memorias de gestión económica.

Además, se pudo contrastar que la diferencia entre municipios más poblados y menos poblados es estadísticamente significativa: los municipios de Castilla y León de más de 50.000 habitantes informan en mayor medida de los recursos colectivos que los municipios más pequeños (de entre 10.000 y 50.000 habitantes).

Por otro lado, se contemplaron elementos relacionados con una sala de prensa municipal. Los resultados corroboran que la actuación del poder municipal en Castilla y León se concreta en la publicación de noticias generalistas y de concesiones, obviando noticias que tienen que ver con la actuación municipal respecto de las cuentas, con la actuación de la oposición en su labor de control del poder, y finalmente de noticias que pudieran contravenir o criticar. No lo publica el poder municipal y la oposición también silencia -aunque quizá lo publique algún medio de comunicación-. Además, dicha actuación es general, independiente del tamaño del municipio estudiado -no se obtuvieron diferencias estadísticamente significativas en los estratos analizados-.

Las limitaciones más importantes son la escasa capacidad de generalización de los resultados al conjunto de España. El ámbito geográfico estudiado, Castilla y León, es una región poco poblada, por lo que no es representativa del conjunto de España, aunque se pueden hacer las oportunas comparaciones en el Mapa Infoparticip@.

http://mapainfoparticipa.com/index/home

Futuros estudios sobre esta cuestión podrían analizar una zona más amplia, o profundizar en aspectos concretos, por ejemplo, el papel de la sala de prensa como elemento propagandístico para el control de la opinión ciudadana, o la vigencia del concepto agenda setting en el ámbito municipal.

Finalmente, aunque este trabajo respeta los principios inspiradores propios de la Alianza para el gobierno abierto de 2011 y de la Ley 19/2013, de 9 de diciembre, de transparencia, acceso a la información pública y buen gobierno (LTBG), también es crítico con algunos aspectos que afectan a la transparencia municipal. Es el caso del punto relativo al control de gobierno municipal por una parte externa o agente exógeno, recogida en el artículo 9 del Capítulo II. Publicidad activa, función adjudicada al Consejo de Transparencia y Buen Gobierno, parece poco realista. Dado el gran volumen de entidades locales sería inviable que un solo organismo 
llevase el control del cumplimiento de las mismas. Este trabajo propone (y así ha estudiado la función de control gubernamental, pero desde otra perspectiva), que sean los grupos políticos municipales de la oposición los encargados también del control directo de los gobiernos locales.

\section{Bibliografía}

Beltrán-Orenes, Pilar; Martínez-Pastor, Esther (2016). “Grado de cumplimiento de las Leyes de transparencia, acceso y buen gobierno, y de reutilización de los datos de contratación de la administración central española". El profesional de la información, v. 25, n. 4, pp. 557-567.

https://doi.org/10.3145/epi.2016.jul.05

Cotino-Hueso, Lorenzo (2015). "Derechos humanos, internet y TICs". En: Rey-Martínez, Fernando (dir.). Los derechos humanos en España: un balance crítico. Valencia: Tirant Lo Blanch, pp. 449-513. ISBN: 9788437097299

España (2007). "Ley 30/2007, de 30 de octubre, de Contratos del sector público". BOE, n. 261, 31 de octubre. Texto consolidado 16/11/2011, en vigor a partir de 16/12/2011.

https://www.boe.es/buscar/act.php?id=BOE-A-2007-18874

España (2013). "Ley 19/2013, de 9 de diciembre, de transparencia, acceso a la información pública y buen gobierno". $B O E$, n. 295, 10 de diciembre. Texto consolidado.

https://www.boe.es/buscar/act.php?id=BOE-A-2013-12887

España (2015). “Ley 18/2015, de 9 de julio, por la que se modifica la Ley $37 / 2007$, de 16 de noviembre, sobre reutilización de la información del sector público". BOE, n. 164, 10 de julio.

https://www.boe.es/diario_boe/txt.php?id=BOE-A-2015-7731

Jurado-Rodas, Basilia (2015). Comunicación institucional y política en las administraciones locales. Tesis doctoral. Universidad de Sevilla.

https://idus.us.es/xmlui/handle/11441/34231

Manfredi-Sánchez, Juan-Luis (2014): “Buenas y malas noticias sobre la Ley de transparencia". Cuadernos de periodistas, 25 de febrero.

http://www.cuadernosdeperiodistas.com/buenas-y-malasnoticias-sobre-la-ley-de-transparencia
Molina-Rodríguez-Navas, Pedro (coord.) (2015). Transparencia de la comunicación pública local: el mapa Infoparticipa. La Laguna (Tenerife): Sociedad Latina de Comunicación Social. ISBN: 9788415968951

http://www.cuadernosartesanos.org/2015/cac78.pdf

Moreno-Sardà, Amparo; Molina-Rodríguez-Navas, Pedro; Corcoy-Rius, Marta (2013). "La información de las administraciones públicas locales. Las webs de los ayuntamientos de Cataluña". Revista latina de comunicación social, n. 68, pp. 502-528.

https://doi.org/10.4185/RLCS-2013-987

Rebolledo, Marta; Rodríguez-Virgili, Jordi; Zamora, Rocío (2015). "La comunicación pública y local: transparencia y participación. Análisis comparativo de las websites municipales de Navarra y Murcia". En: XII Congreso español de ciencia política y de la administración - Aecpa, San Sebastián, 13-15 de julio de 2015.

http://aecpa.es/uploads/files/modules/congress/12/ papers/1281.docx

Rollnert-Liern, Göran (2015). “Ley de transparencia y el derecho de acceso: la discusión doctrinal y parlamentaria en torno a su naturaleza de derecho fundamental". En: CotinoHueso, Lorenzo; Sahuquillo-Orozco, José-Luis; CorredoiraAlfonso, Loreto (eds.). El paradigma del gobierno abierto. Retos y oportunidades de la participación, transparencia y colaboración. Madrid: Universidad Complutense de Madrid, pp. 105-114. ISBN: 9788460696780

http://eprints.ucm.es/35859

Piñar-Mañas, José-Luis (2014). "Transparencia y derecho de acceso a la información pública. Algunas reflexiones en torno al derecho de acceso en la Ley 19/2013, de transparencia, acceso a la información y buen gobierno". Revista catalana de dret públic, n. 49, (diciembre 2014), pp. 1-19. https://doi.org/10.2436/20.8030.01.29

Villoria-Mendieta, Manuel; Jiménez-Sánchez, Fernando (2012), "La corrupción en España (2004-2010): datos, percepción y efectos". REIS. Revista española de investigaciones sociológicas, n. 138, pp. 109-134.

https://doi.org/10.5477/cis/reis.138.109

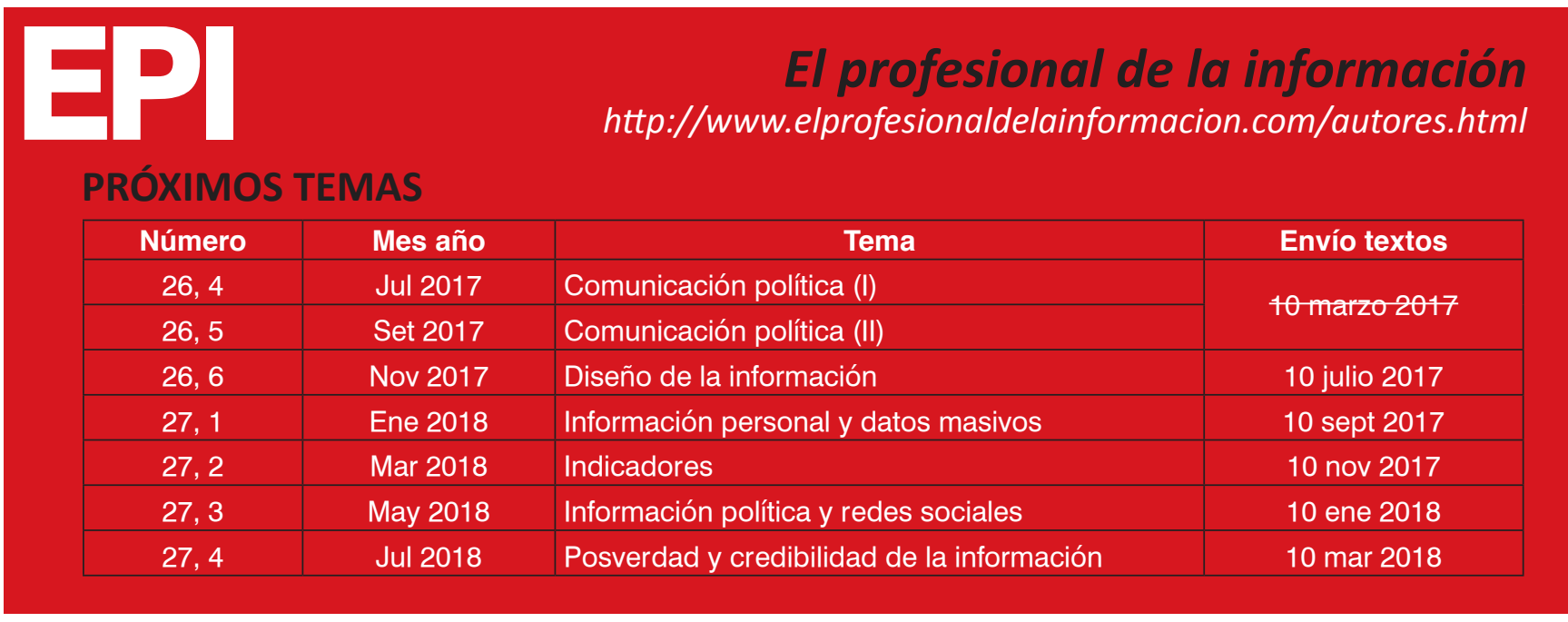

